\title{
Thulium doped ZBLAN fibre ring-cavity amplifier
}

\author{
S Tessarin ${ }^{a^{*}}$, M. Lynch ${ }^{\mathrm{a}}$, J.F. Donegan ${ }^{\mathrm{a}}$, G. Mazé ${ }^{\mathrm{b}}$ \\ ${ }^{a}$ Semiconductor Photonics Group, Physics Department, Trinity College, Dublin 2, Ireland \\ ${ }^{b}$ Le Verre Fluoré, Campus Ker Lann, F-35170 France.
}

\begin{abstract}
We have demonstrated the integration of supporting lasing at $1.88 \mu \mathrm{m}$ for enhancing the gain in a thulium doped ZBLAN amplifier. The laser ring cavity has been created in order to reduce the bottleneck effect caused by the selfterminating nature of the amplifying transition. Two different types of fibre geometry and pumping scheme have been tested and the performance has been compared with a numerical model of the amplifier.
\end{abstract}

Keywords: Thulium doped fibre amplifier, ring-cavity, rate equations model.

\section{INTRODUCTION}

New optical fibre amplifiers are key components in extending the operation of fibre networks to new wavelengths in response to the fast growth of data traffic. Thulium in fluoride fibre is a possible candidate for expanding the WDM operation towards the short-wavelength side of the central $1.53 \mu \mathrm{m}-1.57 \mu \mathrm{m}$ telecom window, due to its fluorescence centered at $1.47 \mu \mathrm{m}$.

Low phonon energy glasses, such as heavy metal fluoride glasses, are crucial for the development of high gain rareearth Thulium fibre amplifiers, since in silica hosts Thulium is affected by strong non-radiative relaxation which reduces the obtainable performance [1]. The realization of an efficient Thulium fluoride fibre amplifier has been prevented by the longer lifetime of the terminating level compared to the upper level of the transition [2].

Several solutions have been proposed in the past to increase the efficiency such as an upconversion pumping scheme [3], where fast depopulation of the terminating level has been achieved by pumping an excited state absorption transition. Another solution is codoping with Holmium where the terminating level is depopulated by resonant transfer to the Holmium neighbor ions [4].

$\mathrm{CW}$ cascade operation at $1.47 \mu \mathrm{m}$ and $1.88 \mu \mathrm{m}$ transition in $\mathrm{Tm}^{3+}$ doped fluoride fibre laser has been extensively studied in the recent years [5][6] and used for achieving simultaneous oscillation at the two wavelengths; this mechanism can be extended to obtain signal amplification in the region $1.45 \mu \mathrm{m}-1.50 \mu \mathrm{m}$. We will present a new amplifier based on the previously mentioned cascade operation.

The amplifier has an all-fibre ring cavity configuration; the feedback for achieving oscillation at $1.88 \mu \mathrm{m}$ is obtained by two Y-couplers with transmission windows centered at $1.9 \mu \mathrm{m}$. A model based on rate equations for the relevant levels has been solved using standard numerical techniques.

\subsection{Spectroscopic Considerations}

The relevant energy levels for trivalent $\mathrm{Tm}^{3+}$ ion in ZBLAN glass are shown in Figure 1, the transition ${ }^{3} \mathrm{H}_{6} \rightarrow{ }^{5} \mathrm{H}_{6}$ centered at $0.79 \mu \mathrm{m}$ is ideal for optical pumping since it has a large absorption cross-section and cheap and reliable laser diodes have already been manufactured at this wavelength.

The transition between the level ${ }^{3} \mathrm{H}_{4}$ and the level ${ }^{3} \mathrm{~F}_{4}$ is responsible for the fluorescence centered at $1.47 \mu \mathrm{m}$ and can be used for amplification, however the former level has a lifetime of $1.6 \mu \mathrm{sec}$ in fluoride fibre, while the terminating level has a lifetime around $10 \mathrm{msec}$. Also from Judd-Oflet calculations the branching ratio for direct remission at $0.8 \mu \mathrm{m}$ is $90 \%$ leaving $8-9 \%$ for the transition at $1.45 \mu \mathrm{m}[7]$.

\footnotetext{
* email: tessaris@tcd.ie; phone: ++353 (0) 16082881.
} 
It is evident that the efficiency of a TDFA is rather low compared to the silica erbium counterpart and substantial population inversion between the two levels involved is difficult to achieve.

In order to improve the steady state population difference between the two states a laser cavity at $1.88 \mu \mathrm{m}$ between the ${ }^{3} \mathrm{H}_{4}$ and the ground state has been provided. The supporting laser oscillation will keep the population of the lower level just above the inversion and reduce the bottleneck effect, allowing higher efficiency for amplification.

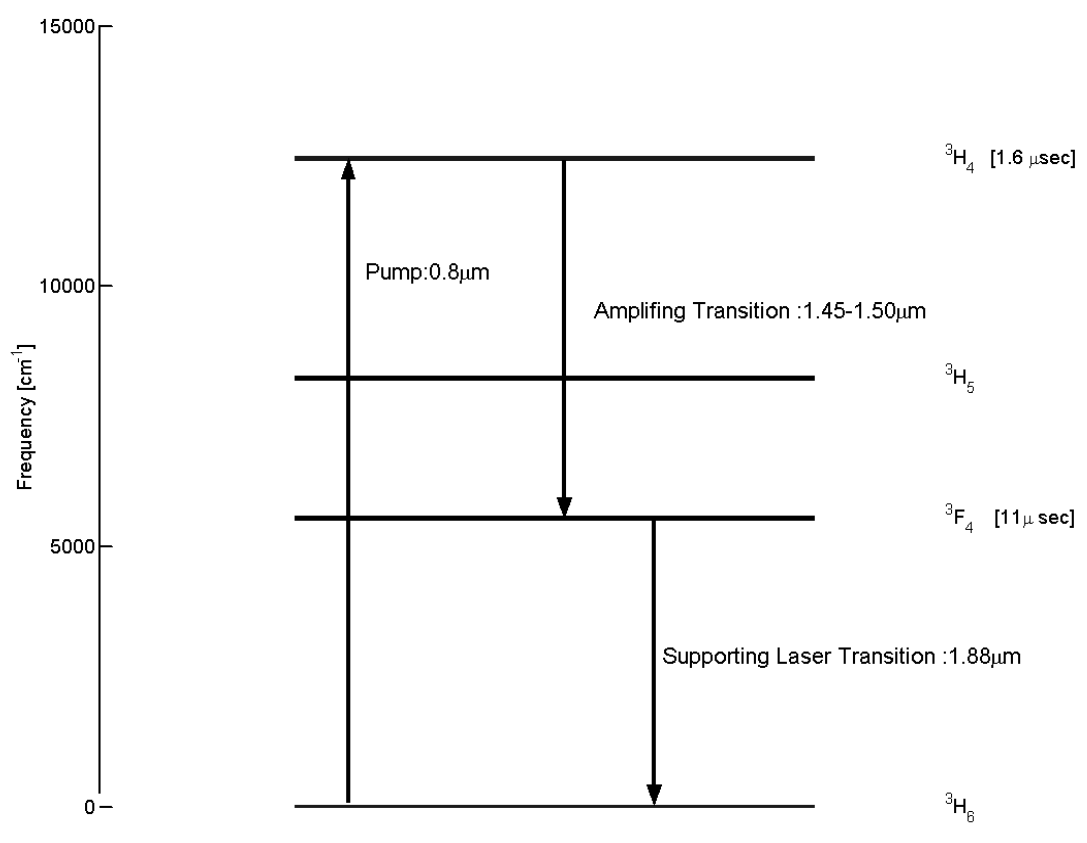

Figure 1: Energy levels of the $\mathrm{Tm}^{3+}$ ions in fluoride glass

The enabling oscillation at $1.88 \mu \mathrm{m}$ has been achieved by embedding the active $\mathrm{Tm}^{3+}$ doped fibre in a ring cavity formed by two chromatic fibre couplers produced from Corning SMF28 fibre.

The fiber chromatic coupler has high transmission centered at $1.9 \mu \mathrm{m}$ and so broad bandwidth reflection around $1.9 \mu \mathrm{m}$ can be obtained, while the second coupler port has high transmission at $1.48 \mu \mathrm{m}$ and partial transmission at $0.800 \mu \mathrm{m}$ for the input and the output of pump and signal.

We have used two different types of fibre with different geometries and lengths; the properties of the fibres are summarized in the table below:

\begin{tabular}{l|lll}
\hline & Unit & Fibre \#1 & Fiber\#2 \\
\hline NA & & 0.234 & 0.17 \\
Core Diameter & $\mu \mathrm{m}$ & 3.2 & 4.4 \\
Length & $\mathrm{mt}$. & 2.4 & 4.0 \\
Cut-off $\lambda$ & $\mu \mathrm{m}$ & 1.05 & 1.0 \\
Doping & p.p.m. & 2000 & 2000 \\
\hline
\end{tabular}


Optical pumping was achieved by a Ti:Sapphire laser tuned to $0.79 \mu \mathrm{m}$. The pump power in the fibre was estimated by taking in account the losses in the couplers and the losses in the fluoride fibre interface. The input signal was provided either by a tunable OPO or an InAlGaAs diode laser with fixed wavelength of $1.49 \mu \mathrm{m}$.

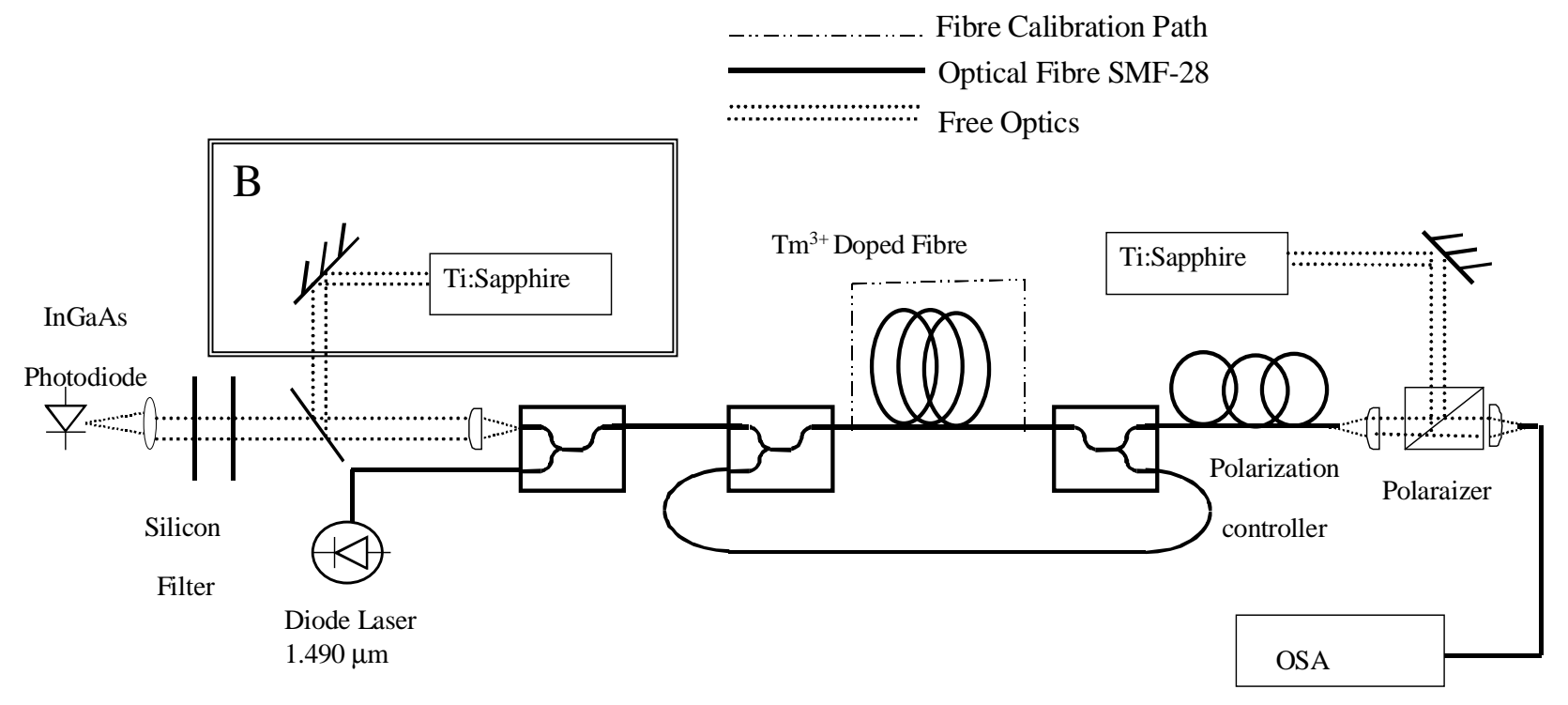

Figure 2: Experimental set up. In box B there is a second Ti:Sapphire that is used only with fibre \#2. Free space optics, and fibre cable have a different line style. The fibre calibration path has been used for the estimation of optical losses.

\section{EXPERIMENT}

The layout of the set-up used is depicted in figure 2, where the difference between the single and double pump configuration is shown, a second Ti:Sapphire ( box B) has been used for the fibre \#2.

An InGaAs photodiode, spectrally tailored for high efficiency at $1.9 \mu \mathrm{m}$, has been used for monitoring the $1.88 \mu \mathrm{m}$ laser power, while a long pass filter in front of the detector, has been placed for filtering wavelengths below $1.5 \mu \mathrm{m}$.

The first measurement which has been carried out was with the shorter fibre \#1 in place. The total power of the fluorescence at $1.9 \mu \mathrm{m}$ has been recorded with the InGaAs detector at different values of pump power while the injected signal with a power of $-40 \mathrm{dBm}$ was launched from the other end of the active fibre (counter-propagating). As shown in fig 3, the laser oscillation started in the cavity when the injected pumping power exceeds $150 \mathrm{~mW}$. In the same configuration, direct gain measurement at $1.49 \mu \mathrm{m}$ has been performed; it shows a gain figure of $6 \mathrm{~dB}$ at $200 \mathrm{~mW}$.

Compared to the case when the external fibre loop has been interrupted, we had observed an extra $4 \mathrm{~dB}$ gain when laser oscillations is established. 


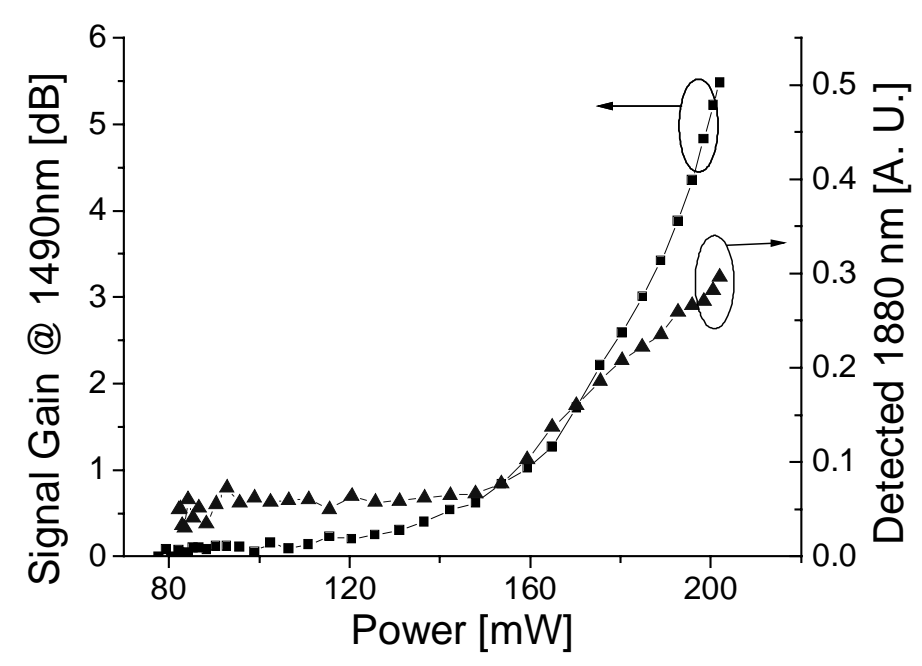

Figure 3: Detected signal gain (left side) and fluorescence at 1880nm (right side), as a function of optical pumping Fibre \#2 has been tested with the aid of a second Ti:Sapphire laser, since higher threshold is expected, the gain increase to a figure over $11 \mathrm{~dB}$ while the threshold recorded for $1.88 \mu \mathrm{m}$ oscillation is around $300 \mathrm{~mW}$ as shown in figure 4 .

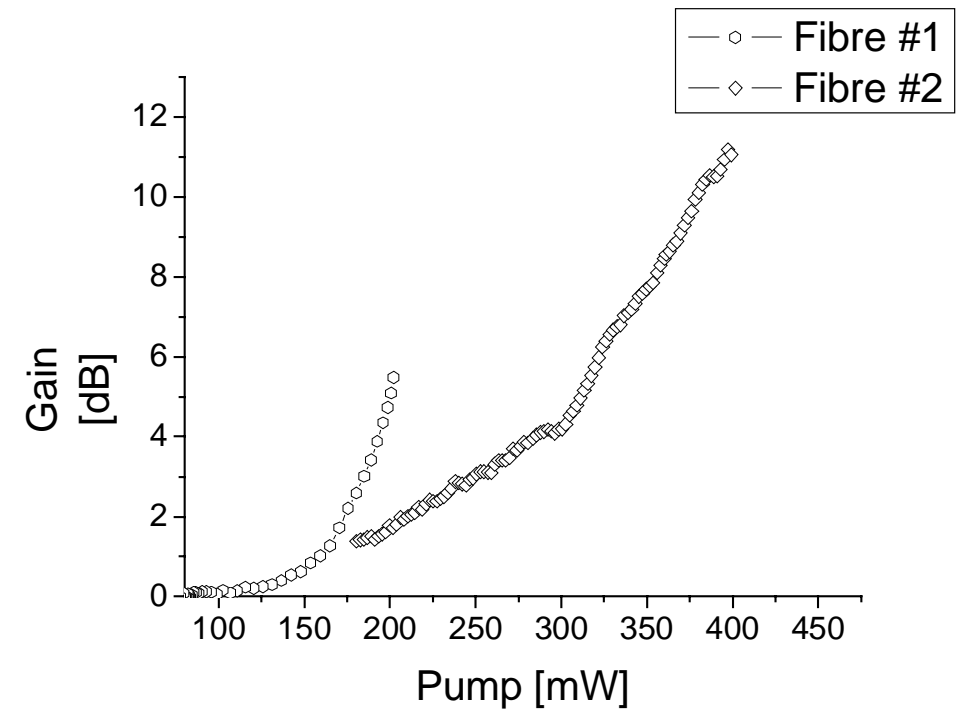

Figure 4 Gain versus pump power for the two fibres

The tuning range has been evaluated for the fibre \#1 with an OPO (Ti:Sapphire pumped). The signal tuned at different wavelengths had a power lower then $-40 \mathrm{dBm}$ in order to remain within the case of small-signal gain. As shown in Figure 5 the gain is relatively flat between $1460 \mathrm{~nm}$ and $1510 \mathrm{~nm}$.

The noise due to the amplified spontaneous emission has been evaluated by the polarization-nulling method [8]. We had rotated the polarization controller until the signal transmission was minimized so half of the power due to the amplified spontaneous emission can be estimated at $0.3 \mathrm{~nm}$ around the signal wavelength.

The noise figure for the signal frequency $v$ and the bandwidth $\Delta v$ is then calculated form the equation (2.1): 


$$
N F=\frac{P_{a s e}}{G h v \Delta v},
$$

where $G$ is the optical signal gain at the frequency $v$.

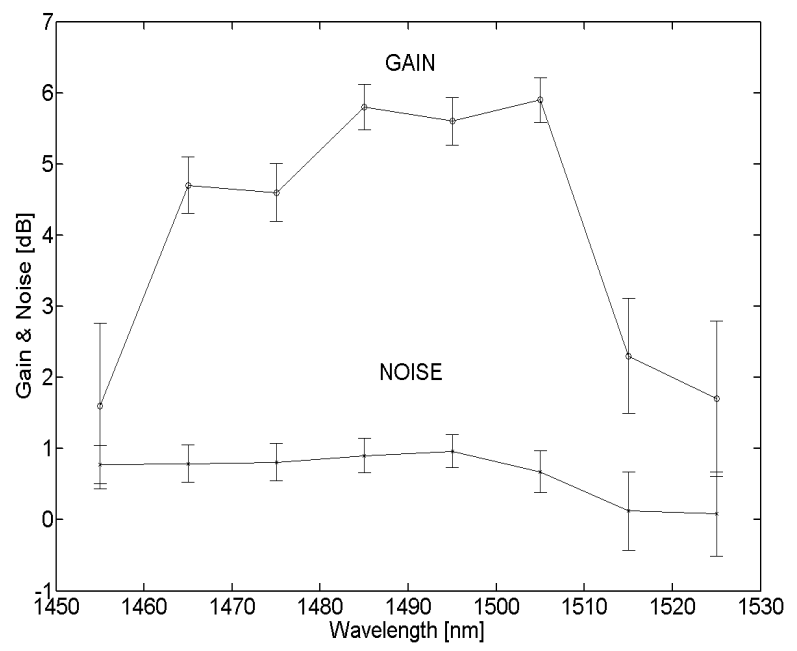

Figure 5: Gain and noise figure vs. signal wavelength for fibre \#1

\section{MODE ANALYSIS}

Mode analysis is a very important tool for design of amplifiers, in our case the transmission fibre (SMF 28) and the active Thulium doped fibre are both single mode at the laser and the signal wavelengths.

The $\mathrm{LP}_{01}$ mode, in the weakly guided approximation, is the only mode supported so the losses due to the fluoride SMF28 interface can be estimated numerically.

This analysis is widely described in the literature [9] [10] and the result is the quasi-guassian electric field $\mathrm{E}_{01}$ calculated for the signal and the laser wavelengths.

The losses at the interface can be calculated by numerical integration over the fibres cross-section A of the two fields involved according to:

$$
\eta=\frac{\left(\int_{0}^{\infty} E_{f} E_{s} d A\right)^{2}}{\left(\int_{0}^{\infty} E_{f}^{2} d A\right)\left(\int_{0}^{\infty} E_{s}^{2} d A\right)}
$$

where $\mathrm{E}_{\mathrm{f}}$ is the $\mathrm{LP}_{01}$ electric field in the fluoride fibre while $\mathrm{E}_{\mathrm{s}}$ is the $\mathrm{LP}_{01}$ electric field in the SMF28 fibre.

The result for the two fibres is shown in table 2 , the value for $1.49 \mu \mathrm{m}$ can be directly compared with the one experimentally observed, since the thulium fibre is transparent at this wavelength. Usually this value strongly depends on the quality of the cleaving and how accurately the two fibres have been joined together and is typically worse than the estimate by a value between 5 and $10 \%$. It can be reduced to a $2-4 \%$ by apply a small amount of index matching fluid between the fibres.

The uncertainty reported for the two test points is the standard deviation of a series of transmission measurements performed each time the two fibres have been cleaved. We have taken this value as the error for the coupling efficiency at $1.88 \mu \mathrm{m}$.

The same procedure can be applied for the determination of the fraction of power of the signal and laser confined in the core respect to the total power that travel in the active fibre. The results are shown in figure 6 and summarized in the table below. 
Table 2: Values for the core confinement factor at the signal end laser wavelngths and extimated losses at the fluoride-trasmission fibres interface.

\begin{tabular}{|l|l|l|}
\hline Integrals: & Fibre\#1 & Fibre\#2 \\
\hline Core Confinement $\%$, Signal $1.49 \mu \mathrm{m}$ & 0.94 & 0.80 \\
\hline Core Confinement \%, Laser $1.88 \mu \mathrm{m}$ & 0.83 & 0.68 \\
\hline Interface Loss \% & $0.92-0.90$ & $0.98-0.96$ \\
\hline
\end{tabular}

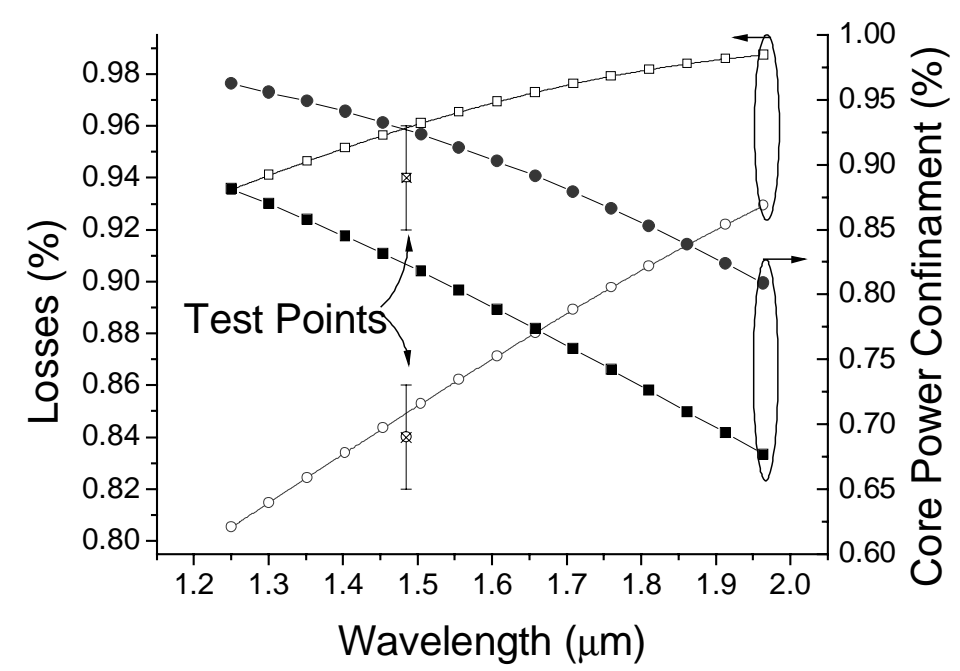

Figure 6: Losses due to the presence of the interface between the fluoride fibre and the transmission fibre (left), $\%$ power of $\mathrm{LP}_{01}$ mode in the core for the two fibres tested, at different wavelengths. The circles are the calculated values for fibre \#1, the squares for fibre \#2.

\section{MODEL}

A steady state model of a TDFA is based on a set of coupled rate equations for the atomic population of the Thulium level involved. The population densities $N_{i}$ of all the relevant levels $\left(\mathrm{i}=1{ }^{3} \mathrm{H}_{6}, \mathrm{i}=2{ }^{3} \mathrm{~F}_{4}, \mathrm{i}=3{ }^{3} \mathrm{H}_{4}\right)$ are subjected to a set of time dependent differential equations and the conservation law. The steady state solution can be algebraically obtain by solving the linear system $d n_{i} / d t=0$ where $n_{i}$ is the atomic population of the level i.

The rate equations for each level are expressed as [11]:

$$
\begin{aligned}
& \frac{d N_{1}}{d t}=-w_{p} N_{1}+w_{l}^{e} N_{2}-w_{l}^{a} N_{1}+A_{31} N_{3}+A_{21} N_{2}, \\
& \frac{d N_{2}}{d t}=-w_{l}^{e} N_{2}+w_{l}^{a} N_{1}-w_{s}^{a} N_{2}+w_{s}^{e} N_{2}+A_{32} N_{3}-A_{21} N_{2}, \\
& \frac{d N_{1}}{d t}=+w_{s}^{a} N_{2}-w_{s}^{e} N_{2}-A_{32} N_{3}-A_{31} N_{3}, \\
& N=\sum_{i=1}^{3} N_{i}
\end{aligned}
$$


where $w_{p}$ is the pump transition rate expressed as:

$$
w_{p}=\frac{\sigma_{a}^{1-3}\left(P_{p}^{+}+P_{p}^{-}\right)}{A_{e f f} h v_{p}} .
$$

The transition rates $w_{p}, w_{p}$ are respectively the absorption (superscript a) and stimulated emission (superscript e) rates at the signal and the laser wavelengths.

$$
\begin{aligned}
& w_{l}^{a, e}=\frac{\sigma_{a, e}^{1-2}\left(P_{l}^{+}+P_{l}^{-}\right)}{A_{e f f} h v_{l}}, \\
& w_{s}^{a, e}=\frac{\sigma_{a, e}^{2-3}\left(P_{s}^{+}+P_{s}^{-}\right)}{A_{e f f} h v_{s}} .
\end{aligned}
$$

With $P_{p, l, s}^{ \pm}$in the equation 4.2 and 4.3 we intended the co (+) and counter(-) propagating power for the pump, laser and signal optical fields (subscript $\mathrm{p}, 1$ and $\mathrm{s}$ ).

The effective area $A_{\text {eff }}$ is calculated via the mode field diameter of the $\mathrm{LP}_{01}$ mode for the laser, signal and pump wavelengths, the absorption and emission cross sections have been mainly taken from previous literature or estimated when no satisfactory value available.

The values are reported in table 3 , together with the spontaneous emission rate $A_{i j}$. The population of the intermediate level ${ }^{3} \mathrm{H}_{5}$ has been taken equal to zero since this level is strongly depopulated by non-radiative relaxation.

Table 3: Values used in the model.

\begin{tabular}{|l|l|l|l|l|}
\hline Parameter & Unit & Symbol & Value & Remarks \\
\hline $\mathrm{Tm}^{3+}$ conc. & $1 / \mathrm{m}^{3}$ & $\rho$ & $1.68 \times 10^{24}$ & Estimated \\
\hline GSA & $\mathrm{m}^{2}$ & $\sigma_{a}^{1-3}$ & $2.8 \times 10^{-25}$ & Measured \\
\hline Signal Emission & $\mathrm{m}^{2}$ & $\sigma_{e}^{2-3}$ & $2.5 \times 10^{-25}$ & {$[13]$} \\
\hline Signal Absorption & $\mathrm{m}^{2}$ & $\sigma_{a}^{2-3}$ & $1.7 \times 10^{-25}$ & {$[14]$} \\
\hline Laser Emission & $\mathrm{m}^{2}$ & $\sigma_{e}^{1-2}$ & $7.1 \times 10^{-25}$ & {$[12]$} \\
\hline Laser Absorption & $\mathrm{m}^{2}$ & $\sigma_{a}^{1-2}$ & $1.2 \times 10^{-25}$ & {$[12]$} \\
\hline Spontaneous rate level 3-1 & $\mathrm{sec}^{-1}$ & $\mathrm{~A}_{31}$ & 1271.9 & {$[14]$} \\
\hline Spontaneous rate level 3-2 & $\sec ^{-1}$ & $\mathrm{~A}_{31}$ & 75.1 & {$[14]$} \\
\hline Spontaneous rate level 2-1 & $\mathrm{sec}^{-1}$ & $\mathrm{~A}_{31}$ & 123.8 & {$[14]$} \\
\hline
\end{tabular}

The evolution of the signal, pump and laser fields in term of optical power in the fibre has to be solved simultaneously with the rate equations [13]: 


$$
\begin{aligned}
& \frac{d P_{p}^{ \pm}}{d z}=\mp \Gamma_{p} \sigma_{a}^{1-3} N_{1}, \\
& \frac{d P_{l}^{ \pm}}{d z}= \pm \Gamma_{l}\left(\sigma_{e}^{1-2} N_{1}-\sigma_{a}^{1-2} N_{2}\right), \\
& \frac{d P_{s}}{d z}=+\Gamma_{s} \sigma_{e}^{3-2} N_{1} .
\end{aligned}
$$

These equations take in account the evolution of the power in the $\mathrm{LP}_{01}$ mode for the pump, signal and laser along the fibre length $\mathrm{z}$. The signs are related to the forward and backward propagation in the cavity. Those optical fields are subject to two boundaries conditions at the start $(\mathrm{z}=0)$ and at the end $(\mathrm{z}=\mathrm{L})$ of the Thulium fibre.

The boundary conditions are expressed as:

$$
\begin{aligned}
& P_{p}^{-}(L)=R_{p} P_{p}^{+}(L), \\
& P_{p}^{+}(0)=R_{p} P_{p}^{-}(0)+P_{p}^{0}, \\
& P_{l}^{-}(L)=R_{l} P_{l}^{+}(L), \\
& P_{l}^{+}(0)=R_{l} P_{l}^{-}(0), \\
& P_{s}(L)=P_{s}^{0}
\end{aligned}
$$

The $R_{p}$ is the reflectivity at both ends of the thulium fibre for the pump wavelength, this value is of the order of $5 \%$ since the contribution of the amount of power that travels through the loop is negligible due to an isolation of $-15 \mathrm{~dB}$. $R_{1}$ is the overall losses of the laser intra-cavity power calculated in section 4 with an extra loss of $-0.2 \mathrm{db}$ due to the chromatic couplers.

The $P_{p}{ }^{0}$ and $P_{s}{ }^{0}$ are the launched power for the pump and for the signal in the case of the counter-propagating pumping scheme, for the fibre \#2 the first equation of 4.5 was modified in order to take into account the second optical pumping at $\mathrm{z}=\mathrm{L}$.

The multimode nature of the pump, where also the higher order mode $\mathrm{LP}_{11}$ is supported, has been evaluated by scaling that estimated in the model with the experimental absorption length observed in term of residual pump at the fibre end. The previous measurements have been performed for a power lower then the threshold in order to avoid that laser oscillation will change the absorption length.

The two parameters are $\Gamma_{p}\left(\right.$ Fibre\#1) $=0.38 \pm 0.02$ and $\Gamma_{p}($ Fibre\# 2$)=0.52 \pm 0.016$, no others parameters have been used.

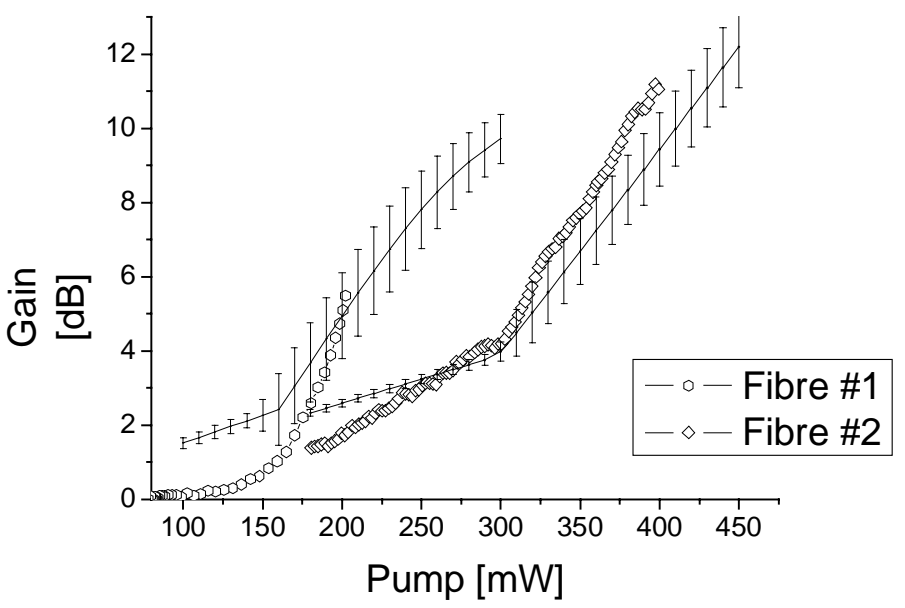

Figure 7:Comparison between the experimental and modeled results. 
The results compared in figure 7 agreed quite well with the experimental data recorded for the two fibres.

The model has been fully written in MATLAB using the function bvp4c [15] for the resolution of two boundary condition problems; a GUI (Figure 8) has been designed to aid the user for creating different simulations.

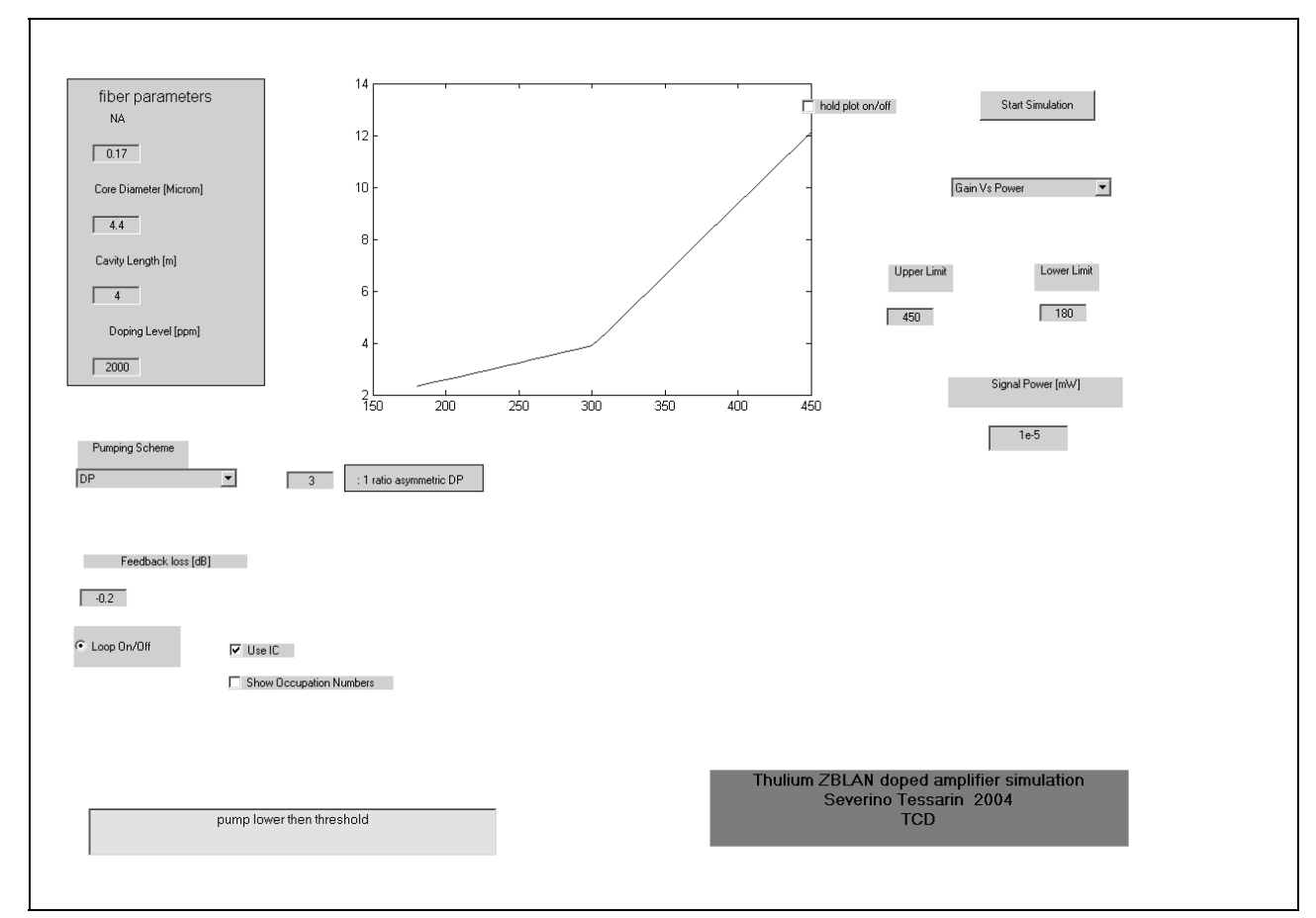

Figure 8: GUI of the model

\section{CONCLUSIONS}

We have demonstrated the enhancement of gain of a Thulium doped ZBLAN amplifier by inclusion of a cavity at 1.88 $\mu \mathrm{m}$. Amplification has been observed in the region 1460-1505 nm, with gain between 6 and $11 \mathrm{~dB}$ for two different cavity constructions. Mode analysis and rate equations modeling are able to describe the behavior of the amplifier in the small-signal gain regime. Further development of the model will permit us to describe the wavelength response and the noise due to amplified spontaneous emission.

\section{REFERENCES}

1. M.J.F. Diggonet, Rare Earth Doped Fibre Lasers and Amplifiers. New York: Marcel Dekker, 1993, ch. 2, pp. 19133.

2. J. Y. Allain, M. Monerie, and H. Poignant, Tunable CW lasing around 0.82, 1.48, 1.88 and 2.35 $\mu$ in thuliumdoped fluorozirconate fibre, Electronics Letters, vol. 25, pp. 1660-1662, 1989

3. T. Komukai, T. Yamamoto, T. Sugawa, Y. Miyajima, $1.47 \mu \mathrm{m}$ band $\mathrm{Tm}^{3+}$ doped fluoride fibre amplifier using a 1.064 um upconversion pumping scheme, Electronics Letters Vol.29, N. 1, 1993, pp. 110-112

4. M. Percival, D. Szebesta, S. T. Davey, N. A. Swain, T. A. King, Thulium sensitized holmium-doped cw fluoride fibre laser of high efficiency, Electronics Letters Vol.28, N.24, 1992, pp.2231-2232

5. M. Percival, D. Szebesta, S. T. Davey, Highly efficient cw cascade operation of $1.47 \mu \mathrm{m}$ and $1.82 \mu \mathrm{m}$ transition in $\mathrm{Tm}^{3+}$ doped fluoride fibre laser, Electronics Letters Vol.28, N.22, 1992, pp. 2063-2065

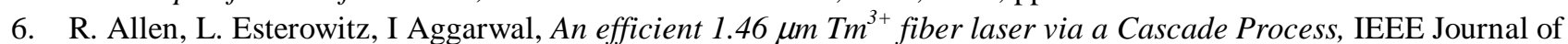
Quantum Electronics, Vol.29, N. 2, 1993, pp. 303-306. 
7. P. Paschotta, P.R. Barber, A.C. Tropper, D.C. Hanna, Characterization and modeling of thulim:ZBLAN upconversion fiber laser, J. Opt. Soc. Am. B, Vol. 14, N. 5 , 1997, pp. 1213-1218.

8. G. Vespasiano, P. Di Cesare, M. Artiglia, and T. Tambosso, Measurement of noise figure in erbium doped fiber amplifiers, presented at Electrotechnical Conference, 1996. MELECON '96., 8th Mediterranean, 1996.

9. J. A. Buck, "Fundamentals of Optical Fibers", Ed. John Wiley \& Sons, 1995, ch.3 ,pp.49-78.

10. J. P. Goure, I Verrier, “Optical Fibre Devices”, IOP Publishing, 2002, ch. 1, pp. 10-15.

11. S. D. Jackson, T. A. King, Theoretical Modelling of Tm-Doped silica Fibre Lasers, J. of Lightwave Tech., Vol. 17, No. 5, 1993,pp. 948-956.

12. R. M. Percival, D. Szebesta, C. P. Seltzer, S. D. Perin, S. T. Davey, and M. Louka, A 1.6- $\mu m$ pumped 1.9- $\mu m$ thulium-doped fluoride fiber laser and amplifier of very high efficiency, IEEE Journal of Quantum Electronics, vol. 31, pp. 489-493, 1995.

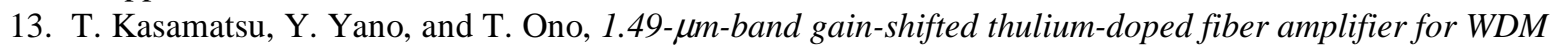
transmission systems, Lightwave Technology, Journal of, vol. 20, pp. 1826-1838, 2002.

14. Sakamoto, T., Spectroscopy of thulium-doped halide glass, in Hewak, D.W. (Ed.), 'Properties, processing and applications of glass and rare earth-doped glasses for optical fibres', EMIS Datareviews Series, no. 22 (Inspec, IEE, Stevenage, UK, 1998), p. 236.

15. http://www.mathworks.com/access/helpdesk/help/techdoc/math/math.html 